\title{
Aneurismas das Artérias Coronárias: uma Rara Manifestação da Doença Renal Policística Autossômica Dominante
}

\author{
Alejandro Sánchez Velásquez', Gustavo T. Gama', J. Ribamar Costa Jr. ${ }^{1}$, \\ Alexandre Abizaid', Fausto Feres ${ }^{1}$
}

\section{RESUMO}

A doença renal policística autossômica dominante (DRPAD), uma das anormalidades genéticas mais comuns, é responsável por $10 \%$ dos casos de insuficiência renal crônica terminal. O acometimento extrarrenal da DRPAD manifesta-se por cistos no fígado e pâncreas, aneurismas intra e extracranianos, e anormalidades cardíacas valvares, comprometendo a raiz da aorta e as valvas aórtica e mitral. Neste artigo descrevemos o caso de um paciente com aneurismas das artérias coronárias no contexto de outras manifestações sugestivas de DRPAD.

DESCRITORES: Aneurisma. Aneurisma coronário. Doenças renais policísticas.

\begin{abstract}
Coronary Aneurysms: a Rare Manifestation of Autosomal Dominant Polycystic Kidney Disease

Autosomal dominant polycystic kidney disease (ADPKD) is one of the most common genetic abnormalities and is responsible for over $10 \%$ of end-stage chronic kidney disease cases. Extrarenal involvement of ADPKD is manifested by liver and pancreatic cysts, intra and extracranial aneurysms and valvular heart disease, involving the aortic root and the aortic and mitral valves. In this article we report a case of a patient with large coronary artery aneurysms in the context of other manifestations suggesting ADPKD.
\end{abstract}

KEY-WORDS: Aneurysm. Coronary aneurysm. Polycystic kidney diseases.

caso de um paciente com grandes aneurismas coronários, no contexto de outras manifestações sugestivas de doença renal policística autossômica dominante. Posteriormente apresentamos uma revisão da literatura sobre a doença renal policística autossômica dominante e suas mais frequentes manifestações cardiovasculares.

\section{RELATO DO CASO}

Trata-se de um paciente do sexo masculino, 59 anos de idade, com antecedente de nefrectomia esquerda e transplante renal em 1995, evoluindo no presente com insuficiência renal não-dialítica (clearance de creatinina $47,6 \mathrm{ml} / \mathrm{min} / 1,73 \mathrm{~m}^{2}$ ). Procurou assistência em nosso serviço em decorrência de quadro de dispneia de caráter progressivo, manifesta no momento da internação aos mínimos esforços, acompanhada de importante edema de membros inferiores. Além da história pregressa de transplante renal, o paciente também já havia sido submetido a troca valvar aórtica com implante de bioprótese em janeiro de 2009, em consequência de quadro de insuficiência aórtica grave. Também já havia sido diagnosticada presença de aneurismas da artéria renal direita, corrigidos durante o transplante renal, e, mais recentemente, aneurismas da aorta 
abdominal $(58 \mathrm{~mm})$ e de ilíacas $(85 \mathrm{~mm}$ à direita e $63 \mathrm{~mm}$ à esquerda) documentados por angiotomografia.

Durante a internação hospitalar, foi constatada disfunção moderada a grave da prótese valvar aórtica, com predomínio de insuficiência, decorrente de fratura na base do folheto, associada a insuficiência mitral grave secundária ao prolapso do folheto anterior e a ruptura de cordoalha tendínea. A função ventricular era preservada (fração de ejeção do ventrículo esquerdo, 62\%). Após a avaliação inicial, foi indicada a retroca da válvula aórtica associada à troca da válvula mitral, ambas por prótese biológica. Por esse motivo, foi submetido a avaliação angiográfica das artérias coronárias. Foram evidenciados aneurismas de grande tamanho em diversos segmentos das três artérias coronárias, sem obstruções significativas (Figuras 1 e 2).

Com o resultado do cateterismo cardíaco, no contexto da doença vascular aórtica, ilíaca e renal, o antecedente de cistos renais e a valvopatia aórtica e mitral, foi estabelecido o diagnóstico presuntivo de doença renal policística autossômica dominante.

No planejamento terapêutico, estabeleceu-se que a correção dos aneurismas arteriais periféricos seria realizada em uma segunda etapa, após o tratamento das afecções valvares. Foi considerada, como primeira opção, a correção cirúrgica, uma vez que a anatomia do aneurisma da artéria ilíaca direita ofereceria dificuldades ao tratamento percutâneo.

Em abril de 2010 foi submetido a cirurgia valvar com implante de válvulas Biocor ${ }^{\mathrm{TM}}$ (St. Jude Medical, St. Paul, Estados Unidos) em posição aórtica e mitral, com sucesso. Evoluiu no pós-operatório com bloqueio atrioventricular total, necessitando marca-passo provi-

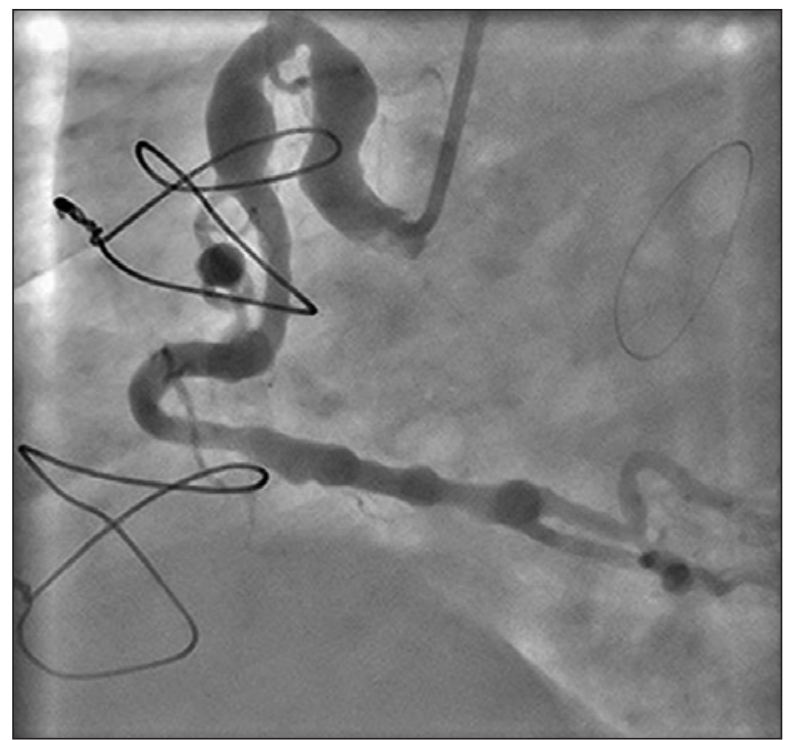

Figura 1 - Artéria coronária direita, em projeção oblíqua anterior esquerda, apresentando grandes e múltiplos aneurismas. sório, hemorragia digestiva alta por úlcera de estresse e agudização da insuficiência renal crônica. Ecocardiogramas seriados realizados no período pós-operatório demonstraram que as próteses se mantiveram normofuncionantes, mas com piora da função ventricular (fração de ejeção do ventrículo esquerdo, 39\%). O paciente desenvolveu choque misto (cardiogênico e séptico) de predomínio distributivo, secundário a sepse por foco pulmonar (pneumonia associada a ventilação mecânica prolongada). No 29ㅇ dia de evolução pós-operatória, o paciente apresentou parada cardiorrespiratória em atividade elétrica sem pulso, não responsiva às manobras de ressuscitação cardiopulmonar.

\section{DISCUSSÃO}

A doença renal policística autossômica dominante é uma das anormalidades genéticas mais comuns na atualidade e tem grande variabilidade inter e intrafamiliar. Acomete um a cada mil indivíduos e é responsável por $10 \%$ dos casos de insuficiência renal crônica terminal, que tipicamente se desenvolve entre a quarta e a sexta décadas de vida. Embora assintomática na maioria dos pacientes, as manifestações extrarrenais da doença renal policística autossômica dominante podem se tornar clinicamente relevantes com o aumento da expectativa de vida dos pacientes. Elas se manifestam por cistos no fígado e pâncreas, anormalidades no coração e vasos sanguíneos ou uma combinação desses sítios. ${ }^{7}$

A maior parte das anormalidades renais e extrarrenais encontradas é consistente com um defeito generalizado na diferenciação das células epiteliais e/ou na função da matriz extracelular como a expressão primária da anormalidade genética dessa doença. ${ }^{8}$

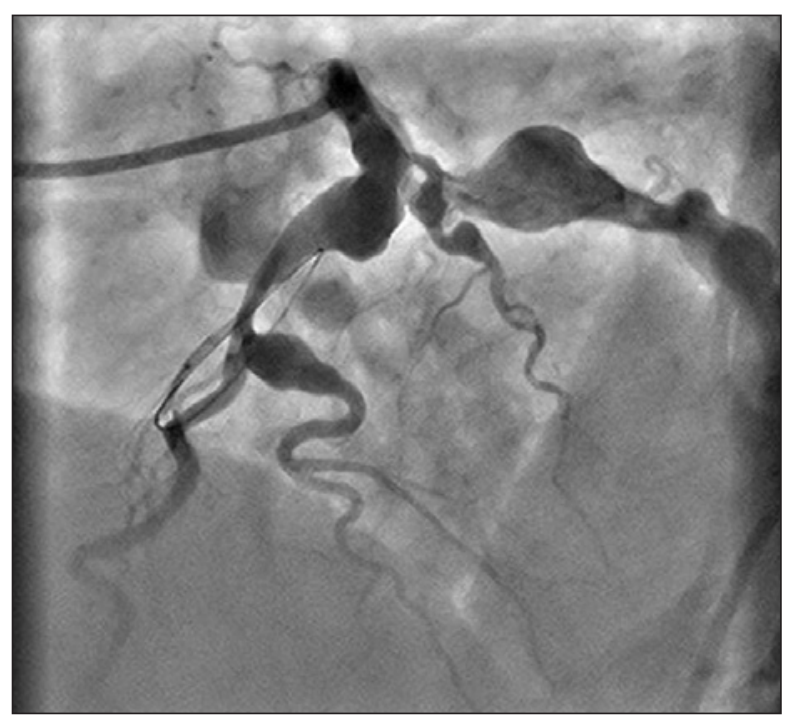

Figura 2 - Artéria coronária esquerda, em projeção oblíqua anterior esquerda, apresentando grandes e múltiplos aneurismas na descendente anterior e na circunflexa. 
O acometimento cardiovascular é uma das maiores causas de morbidade e mortalidade nesses pacientes. Aneurismas intracranianos são quatro a cinco vezes mais frequentes em pacientes com doença renal policística autossômica dominante que na população geral. ${ }^{9}$ Aneurismas extracranianos acometendo artérias coronárias, aorta, artéria renal e esplênica também têm sido relatados. Anormalidades cardíacas valvares são frequentes na doença renal policística autossômica dominante, comprometendo raiz da aorta, ânulo e valvas aórtica e mitral. O prolapso da valva mitral é a afecção valvar mais comum, acometendo até $25 \%$ dos pacientes. ${ }^{10}$

Os relatos de aneurismas coronários associados à doença renal policística autossômica dominante são limitados a três relatos de $\operatorname{caso}^{3,4,6}$ e à descrição de uma pequena série de pacientes ${ }^{5}$ e não existem recomendações específicas para seu tratamento nesse cenário.

Atualmente sabemos que os aneurismas coronários são, em sua maioria, assintomáticos, sendo descobertos na investigação de outras afecções ou em exames de rotina. Quando os sintomas ocorrem, angina ou infarto são as características mais comuns de apresentação. O infarto do miocárdio é a apresentação inicial em $30 \%$ a $50 \%$ dos casos. ${ }^{11,12}$ Um estudo pós-morte publicado na década do 1960 relatou a presença de trombos em 7 de cada 10 pacientes com aneurismas coronários. ${ }^{13}$ Vários relatos de caso também demonstraram infarto do miocárdio decorrente de trombose de aneurismas coronários sem lesões obstrutivas. ${ }^{14,15}$ Swanton et al. ${ }^{16}$ demonstraram redução do fluxo sanguíneo coronário em pacientes com aneurismas coronários, levando à formação de coágulos em seu interior. Aneurismas podem complicar-se com ruptura, evento raro, sem ocorrências nos 978 pacientes com aneurismas na base de dados CASS. ${ }^{11}$

O tratamento dos aneurismas coronários envolve terapia de antiagregação plaquetária e anticoagulação. ${ }^{17}$ Aneurismas responsáveis por isquemia miocárdica têm sido tratados com stents recobertos por enxertos autólogos de veia safena ou implantes de Jostent ${ }^{\mathrm{TM}}$ (Jomed $A B$, Helsimburgo, Suécia). ${ }^{18-21}$ Esses tratamentos têm sido descritos somente em relatos de caso e não são validados por investigações científicas controladas. Os aneurismas muito grandes e/ou multiarteriais têm sido tratados com enxertos vasculares coronários. ${ }^{22}$

\section{CONFLITO DE INTERESSES}

Os autores declararam inexistência de conflito de interesses relacionado a este manuscrito.

\section{REFERÊNCIAS}

1. Gziut Al, Gil RJ. Coronary aneurysms. Pol Arch Med Wewn. 2008;118(12):741.

2. Villines TC, Avedissian LS, Elgin EE. Diffuse nonatherosclerotic coronary aneurysms: an unusual cause of sudden death in a young male and a literature review. Cardiol Rev. 2005; 13(6):309-11.
3. Christ M, Bechtel U, Schnaack S, Theisen K, Wehling $M$. Aneurysms of coronary arteries in a patient with adult polycystic kidney disease: arteriosclerosis or involvement by the primary disease? Clin Investig. 1993;71(2):150-2.

4. Adubofour K, Sidaway L, Glatter T. Coronary artery aneurysms in association with adult polycystic kidney disease. Am Heart J. 1994;127(5):1411-3.

5. Hadimeri H, Lamm C, Nyberg G. Coronary aneurysms in patients with autosomal dominant polycystic kidney disease. J Am Soc Nephrol. 1998;9(5):837-41.

6. Kucukdurmaz Z, Buyukhatipoglu H, Sezen Y, Kaya Z. Polycystic kidney disease with coronary aneurysm and acute coronary syndrome. Intern Med. 2009;48(22):1989-91.

7. Grantham JJ. Clinical practice. Autosomal dominant polycystic kidney disease. N Engl J Med. 2008;359(14):1477-85.

8. Watson ML. Complications of polycystic kidney disease. Kidney Int. 1997;51(1):353-65.

9. Pirson $Y$, Chauveau D, Torres V. Management of cerebral aneurysms in autosomal dominant polycystic kidney disease. J Am Soc Nephrol. 2002;13(1):269-76.

10. Lumiaho A, Ikäheimo R, Miettinen R, Niemitukia L, Laitinen $\mathrm{T}$, Rantala A, et al. Mitral valve prolapse and mitral regurgitation are common in patients with polycystic kidney disease type 1 . Am J Kidney Dis. 2001;38(6):1208-16.

11. Swaye PS, Fisher LD, Litwin P, Vignola PA, Judkins MP, Kemp HG, et al. Aneurysmal coronary artery disease. Circulation. 1983;67(1):134-8.

12. Wang KY, Ting CT, St John Sutton $M$, Chen YT. Coronary artery aneurysms: a 25-patient study. Catheter Cardiovasc Interv. 1999;48(1):31-8.

13. Daoud A, Pankin D, Tulgan H, Florentin R. Aneurysms of the coronary artery: report of ten cases and review of the literature. Am J Cardiol. 1963;11:228-37.

14. Rath S, Har-Zahav Y, Battler A, Agranat O, Rotstein Z, Rabinowitz B, et al. Fate of nonobstructive aneurysmatic coronary artery disease: angiographic and clinical follow-up report. Am Heart J. 1985;109(4):785-91.

15. Myler RK, Scheshtmann NS, Rosenblum J, Collinsworth KA, Bashour TT, Ward K, et al. Multiple coronary artery aneurysms in an adult associated with extensive thrombus formation resulting in myocardial infarction: successful treatment with intracoronary urokinase, intravenous heparin, and oral anticoagulation. Cathet Cardiovasc Diagn. 1991;24(1):51-4.

16. Swanton RH, Thomas ML, Coltart DJ, Jenkins BS, Webb-Peploe MM, Williams BT. Coronary artery ectasia: a variant of occlusive coronary arteriosclerosis. Br Heart J. 1978;40(4):393-400.

17. Syed M, Lesch M. Coronary artery aneurysm: a review. Prog Cardiovasc Dis. 1997;40(1):77-84.

18. Chen MF, Chien KL, Tsang YM, Liau CS, Lee YT. Transcatheter embolization in treatment of congenital coronary artery aneurysm. Am Heart J. 1996;131(2):396-7.

19. Heuser RR, Woodfield S, Lopez A. Obliteration of a coronary artery aneurysm with a PTFE-covered stent: endoluminal graft for coronary disease revisited. Catheter Cardiovasc Interv. 1999; 46(4):113-6.

20. Antonellis IP, Patsilinakos SP, Pamboukas CA, Kranidis AJ, Prappa E, Filippatos G, et al. Sealing of coronary artery aneurysm by using a new stent graft. Catheter Cardiovasc Interv. 1999;48(1):96-9.

21. Kereiakes DJ, Broderick TM, Howard WL, Anderson LC, Weber M, Mitts DL. Successful long-term therapy following saphenous vein-covered stent deployment for atherosclerotic coronary aneurysm. Catheter Cardiovasc Interv. 2002;55(1):100-4.

22. Harandi S, Johnston SB, Wood RE, Roberts WC. Operative therapy of coronary arterial aneurysm. Am J Cardiol. 1999; 83(8):1290-3. 